\title{
CONDICIONES DE CULTIVO ESTÁNDAR RELACIONADOS CON LA PRODUCCIÓN DE ASTAXANTINA EN HAEMATOCOCCUS PLUVIALIS
}

\section{STANDARD CULTURE CONDITIONS RELATED TO THE PRODUCTION OF ASTAXANTHIN IN HAEMATOCOCCUS PLUVIALIS}

\author{
*Camacho K. Judith E., Lancheros D. Ana G., Huerfano T. Myriam. J. \\ Universidad Colegio Mayor de Cundinamarca, Facultad Ciencias de la salud Calle 28 No 6- 02 Bogotá \\ -Colombia
}

Recibido 21 de Octubre 2015; aceptado 30 de Marzo de 2016

\section{RESUMEN}

La astaxantina es un pigmento natural ampliamente distribuido en la naturaleza y de gran interés comercial como colorante y por sus diversas propiedades como pigmento $y$ bioactivas. El Haematococcus pluvialis es un alga verde que acumula carotenoides, principalmente astaxantina cuando es expuesto a condiciones de estrés. Sin embargo, en la literatura no se encuentran estudios sistemáticos que permitan determinar las condiciones de estrés que mejor favorezcan la acumulación de astaxantina para una producción industrial más rentable. Para tal fin se debe establecer inicialmente la línea base de comportamiento de $\mathrm{H}$. pluviales a condiciones de cultivo estándar, propósito del presente trabajo mediante la revisión teórica de las diferentes condiciones de crecimiento de $\mathrm{H}$. pluviales en los medios de 
Autor a quien dirigirse la correspondencia. *Camacho Kurmen Judith Elena. Correo electrónico: jelenacamacho@hotmail.com. cultivo reportados relacionados con la mayor producción de astaxantina, para lo cual se tienen en cuenta valores de $\mathrm{pH}$, temperatura, $\mathrm{CO}_{2}$, aireación, luz, ciclos luz/oscuridad, agitación, deficiencia de nitrógeno y fósforo.

Palabras claves: Pigmento, microalga, carotenoides, condiciones del cultivo, medio de cultivo

\section{ABSTRACT}

Astaxanthin is a natural pigment widely distributed in nature and of great commercial interest as a colorant and its various properties as a pigment and bioactive. Haematococcus pluvialis is a green alga which accumulates carotenoids, particularly astaxanthin when exposed to stress conditions. However, in the literature there are systematic studies to determine the stress conditions that best the accumulation of astaxanthin for more profitable industrial production. To that end should initially set the base line behaviour of $H$. pluvialis in standard culture conditions, purpose of this work was the reviewing of the different growth conditions of $H$. pluvialis in the culture media reported related with more production of astaxanthin, for which takes into account $\mathrm{pH}$, temperature, $\mathrm{CO}_{2}$, aeration, light, light / dark cycles, agitation, nitrogen and phosphorus deficiency.

Keywords: carotenoids, microalgae, culture conditions, the culture medium, pigment.

\section{INTRODUCCIÓN}

Las microalgas son fuente de un gran número de compuestos bioactivos de interés industrial, como los carotenoides que se utilizan como colorantes naturales en alimentación animal y humana, así como en 
la industria farmacéutica, cosmética y en la acuicultura. Además se han propuesto como agentes efectivos en la prevención de una variedad de enfermedades, debido a su capacidad antioxidante, inmunoreguladora, anti-inflamatoria y anti-cancerígena.

El ketocarotenoide astaxantina es el más importante desde el punto de vista biotecnológico. Hoy la mayor cantidad de astaxantina es producida por síntesis química y es vendida a un precio de US $\$ 2500 / \mathrm{kg}$. El alto precio y el incremento en la demanda para este compuesto, especialmente de origen natural, en las diferentes industrias, hace que sea de interés la producción astaxantina a partir de microalgas como la $H$. pluvialis, que acumula cantidades importantes (más del $4 \% / g$ de peso seco) y de mejor calidad que las obtenidas por otras fuentes como levaduras y plantas. La acumulación del

pigmento en $H$. pluvialis ocurre durante la transformación de la microalga desde el estado vegetativo (fase verde) a aplanospora (fase roja) cuando cesa su crecimiento en la fase estacionaria. Otros tipos de estrés inducen la acumulación de astaxantina como son temperatura, intensidad lumínica, ciclos de luz/oscuridad, concentración de nutrientes, $\mathrm{pH}$, especies reactivas de oxígeno, sales y presencia de inhibidores de procesos metabólicos a diferente nivel. Sin embargo, esta microalga tiene sus dificultades en el momento de cultivo y de obtener el pigmento en cantidades de interés debido a su ciclo celular complejo. Es por lo tanto necesario establecer las condiciones de crecimiento adecuadas que lleven a una buena producción de biomasa y posteriormente de astaxantina para poder considerar a $H$. pluvialis como fuente natural de este.

\section{MATERIALES Y MÉTODOS}

Condiciones de cultivo. Microorganismo estudiado: Haematococcus pluvialis.

Establecimiento de crecimiento a condiciones estándar reportadas para establecer una plataforma de trabajo y línea base de crecimiento.
Revisión de artículos relacionados con el cultivo de $H$. pluvialis bajo condiciones estándar y controladas, reportadas. Los parámetros elegidos para seleccionar los medios de cultivos y condiciones relacionadas fueron la biomasa por peso seco y la concentración de astaxantina. 
Determinación de la producción de astaxantina.

Se determinó la producción de la concentración de astaxantina y unidades de medición.

\section{Diseño experimental y análisis de datos}

Se realizó un análisis multicriterio de los medios reportados en la literatura y de condiciones fotoautotróficas: $\mathrm{pH}$, temperatura, $\mathrm{CO}_{2}$, aireación, luz, ciclos luz/oscuridad, agitación, deficiencia de nitrógeno.

A y Gyun Lee, 2006), pH y nutrientes orgánicos como acetato (Eonseon et. al.,

\section{RESULTADOS Y DISCUSIÓN}

Fisiológicamente la acumulación de astaxantina en $H$. pluvialis ocurre en respuesta a varias condiciones de estrés ambiental como alta intensidad de luz, limitaciones de nitrógeno, fósforo y estrés por sal. (Steinbremer J. y Linden H, 2001) (Wang et al., 2013).

El cambio morfológico en $H$. pluvialis de células vegetativas verdes, para incrementar el número de células, a células con quistes rojos que acumulan astaxantina, es inducido por algunos factores, tales como: temperatura alta, deficiencia de nutrientes (nitrato, magnesio, sulfato y fosfato), alta intensidad de luz, alta salinidad y estrés oxidativo. (Yoshimura et. al., 2006) (Ranbjar et. al, 2008) (Hana et. al. 2013).

Otras de las condiciones de estrés ensayadas son: estrés de nutrientes, alta intensidad de luz, alta salinidad (Lababpour
2006) ó combinación cloruro de sodio/acetato de sodio, aumentando el contenido total de carotenoides y contenido total de astaxantina. (Vidhyavathi et al., 2008).

En la tabla 1 se pueden observar en forma general las diferentes condiciones de cultivo ensayadas en los estudios revisados, incluyendo el uso de fotobioreactores, mezclándolo con irregulares ciclos de luz/oscuridad. (Ranbjar et. al., 2008).

Tabla 1. Condiciones de cultivos de haematococcus pluvialis relacionados con concentración de astaxantina.

\begin{tabular}{|l|l|}
\hline $\begin{array}{l}\text { CONDICIONES DE CULTIVO } \\
\text { AUTOR }\end{array}$ & $\begin{array}{l}\text { CONCENTRACION } \\
\text { DE ASTAXANTINA }\end{array}$ \\
\hline $\begin{array}{l}\text { Se usó disminución de } \\
\text { nitrato (5\% del medio), alta }\end{array}$ & Acumulación \\
intensidad de luz blanca & \\
$\left(150 \mathrm{umol} / \mathrm{m}^{2} \mathrm{~s}\right)$ Grünewald & \\
et al.,2000 & \\
\hline
\end{tabular}


@.LIMENTECH CIENCIA Y TECNOLOGÍA ALIMENTARIA ISSN 1692-7125. Volumen 14 No. 1, p. 70 -80, año 2016 Facultad de Ingenierías y Arquitectura Universidad de Pamplona

\begin{tabular}{|c|c|}
\hline $\begin{array}{l}\text { Presencia de acetato de } \\
\text { sodio ( } 45 \mathrm{mM}), \mathrm{Fe}(\mathrm{II})(450 \\
\mathrm{uM}) \text {, alta intensidad de luz } \\
(125 \quad \text { umol/m²s). } \\
\text { Steinbremer J y Linden H } \\
2001\end{array}$ & $\begin{array}{l}13,5 \mathrm{mg} / \mathrm{g} \text { peso } \\
\text { seco }\end{array}$ \\
\hline $\begin{array}{l}\text { Medio bristol, temperatura } \\
25^{\circ} \mathrm{C} \text {, fotoperiodo } \\
12 / 12 / 1.25 \mathrm{~g} / \mathrm{l} \text { de } \mathrm{NaNO}_{3} \text { es } \\
\text { la concentración adecuada } \\
\text { para un mayor rendimiento } \\
\text { e biomasa; } 2003 \text {. }\end{array}$ & Acumulación \\
\hline 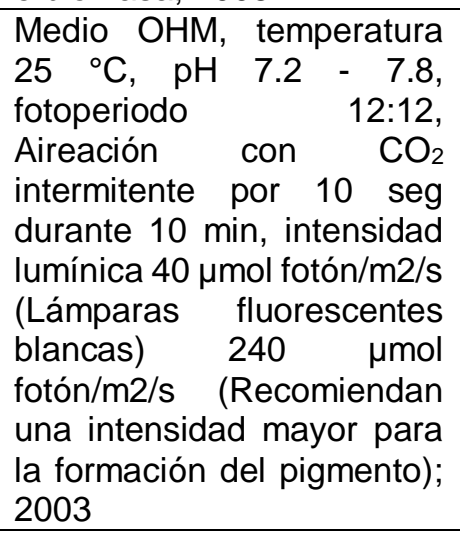 & 9,6 mg l-1 día \\
\hline $\begin{array}{l}\text { Se usó alta intensidad de } \\
\text { luz }(350 \text { umol/m²s }) \text { Wang } B \text {. } \\
\text { y Zarca } A, 2003\end{array}$ & $43 \mathrm{mg} / \mathrm{ml}$ \\
\hline $\begin{array}{l}\text { Medio bristol, Temperatura } \\
23+/-2 \text { durante } 14 \mathrm{~d}, \mathrm{pH} \\
6.0 \text {, fotoperiodo } 12 / 12, \mathrm{PFD} \\
35 \mathrm{Y} 85 \mathrm{~mol} \mathrm{~m}-2 \mathrm{~s}-, \mathrm{NH} 4 \mathrm{Cl} \\
\text { como fuente de nitrógeno } \\
\text { que obtuvo la mayor } \\
\text { concentración de biomasa; } \\
\text { Cifuentes et al. } 2003\end{array}$ & $10,3 \mathrm{mg} / \mathrm{g}$ \\
\hline $\begin{array}{l}\text { Se usa estrés oxidativo: } \\
\text { acetato de sodio, Fe(II), alta } \\
\text { intensidad de luz Wang et } \\
\text { al., 2004. }\end{array}$ & $\begin{array}{c}\text { Acumulación de } \\
\text { astaxantina }\end{array}$ \\
\hline $\begin{array}{lr}\text { Medio Basal Bold, } \\
\text { Temperatura } & 28 \\
\text { oC,lluminacion continua, } 1.5 \\
\text { \% CO2, BAR para mayor } \\
\text { producción de astaxantina); } \\
\text { Domínguez et al., 2004. }\end{array}$ & $98 \mathrm{mg} / \mathrm{g}$ \\
\hline $\begin{array}{l}\text { Se usó luz blanca LEDS } \\
\left(600 \text { umol } / \mathrm{m}^{2} \mathrm{~s}\right) \text { Torzillo et } \\
\text { al., } 2005 .\end{array}$ & $\begin{array}{l}23,7 \% \text { del total } \\
\text { de carotenoides }\end{array}$ \\
\hline $\begin{array}{l}\text { Se usó alta intensidad de } \\
\left.\text { luz ( } 80 \mathrm{umol} / \mathrm{m}^{2} \mathrm{~s}\right), 5 \% \mathrm{CO} 2, \\
\text { limitación nitrógeno y } \\
\text { fósforo; Kang et al., } 2006 \text {. }\end{array}$ & $77 \mathrm{mg} / \mathrm{g}$ \\
\hline
\end{tabular}

\begin{tabular}{|c|c|}
\hline $\begin{array}{l}\text { Se usó lluminación con } \\
\text { LEDS azul (8-12 umol/m²s), } \\
\text { ajuste de } \mathrm{pH} \text { menor a 9,5; } \\
\text { Labapour et al., } 2005 \text {. }\end{array}$ & $50-70 \mathrm{ug} / \mathrm{ml}$ \\
\hline $\begin{array}{l}\text { Alta intensidad de luz, pH } \\
6.3 \text { +/- } 0.5, \mathrm{CO} 2 \text {, agitación; } \\
\text { Lababpour A. y Gyun Lee, } \\
2006\end{array}$ & $12 \mathrm{mg} / \mathrm{ml}$ \\
\hline $\begin{array}{l}\text { Luz azul LEDS (12 } \\
\text { umol/m²s); Yoshimura et.al, } \\
2006\end{array}$ & $\begin{array}{l}0,13-0,16 \\
\mathrm{ug} / \mathrm{ml} / \mathrm{h}\end{array}$ \\
\hline $\begin{array}{l}\text { Se usó alta intensidad de } \\
\text { luz ( } 430 \text { umol } / \mathrm{m}^{2} \mathrm{~s} \text { ), alta } \\
\text { concentración de cloruro de } \\
\text { sodio (más de } 0,8 \% \text { ), estrés } \\
\text { de nitrógeno y fósforo, } \\
\text { presencia de nitrato de } \\
\text { calcio, acetato de sodio ( } 45 \\
\mathrm{mM} \text { ), sulfato de hierro ( } 450 \\
\text { uM) y malonato; } \\
\text { Eonseon et al.,2006 }\end{array}$ & $\begin{array}{l}\text { Alta producción } \\
\text { de astaxantina }\end{array}$ \\
\hline $\begin{array}{l}\text { Se usó temperatura (25ㅇ), } \\
\text { aireación de CO2 ( } 1,5 \% \mathrm{v} / \mathrm{v}) \text {, } \\
\text { alta intensidad de luz blanca } \\
(75 \text { umol/m²s), pH menor de } \\
8.0 \text {, fertilizantes (urea + } \\
\text { K2HPO4+KH2PO4 ó N-P- } \\
\text { K); Meltem et al.,2007 }\end{array}$ & $\begin{array}{ll}\begin{array}{l}\text { Biomasa } \\
\mathrm{g} / \mathrm{L}\end{array} & 0,90 \\
\end{array}$ \\
\hline $\begin{array}{l}\text { Se uso alta intensidad de } \\
\left.\text { luz ( } 700 \text { umol } / \mathrm{m}^{2} \mathrm{~s}\right), 2,42 \mathrm{~g} / \mathrm{L} \\
\text { de trisacetato; Steinbrenner } \\
\text { J. y Sandmann G., } 2006 \text {. }\end{array}$ & $\begin{array}{l}11,4 \mathrm{mg} / \mathrm{g}+/- \\
0,9 \text { peso seco }\end{array}$ \\
\hline $\begin{array}{l}\text { Medio OHM, } \mathrm{pH} 7.0, \\
\text { temperatura } 25 \pm 0,5{ }^{\circ} \mathrm{C} \text {, } \\
\text { lámparas fluorescentes } \\
\text { marca Sylvania Daylight } \\
\text { F48T12/D de } 39 \mathrm{~W} \text { con una } \\
\text { intensidad lumínica de } 5000 \\
\pm 50 \text { lux, 0.51\% CO2, } \\
\text { concentración nitrógeno } 4 \\
\mathrm{mM} \text {; Domínguez et al,. } \\
2007 \text {. }\end{array}$ & Acumulación \\
\hline 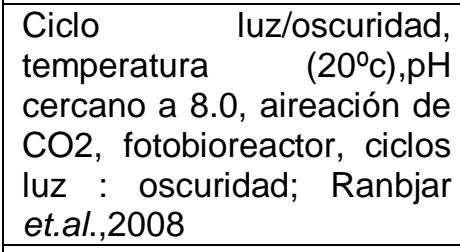 & $\begin{array}{c}460-600 \\
\mathrm{mg} / \mathrm{ml}\end{array}$ \\
\hline $\begin{array}{l}\text { Presencia de alta intensidad } \\
\left.\text { de luz ( } 60 \mathrm{umol} / \mathrm{m}^{2} \mathrm{~s}\right) \text {, estrés } \\
\text { de nutrientes, cloruro de } \\
\text { sodio( } 17,1 \mathrm{mM}) / \text { acetato de } \\
\text { sodio }(4,4 \mathrm{mM}) \text { Vidhyavathi } \\
\text { et al., } 2008 .\end{array}$ & $\begin{array}{c}24.5-32 \mathrm{mg} / \mathrm{g} \\
\text { peso seco }\end{array}$ \\
\hline
\end{tabular}


@.LIMENTECH CIENCIA Y TECNOLOGÍA ALIMENTARIA ISSN 1692-7125. Volumen 14 No. 1, p. 70 -80, año 2016 Facultad de Ingenierías y Arquitectura Universidad de Pamplona

\begin{tabular}{|c|c|}
\hline 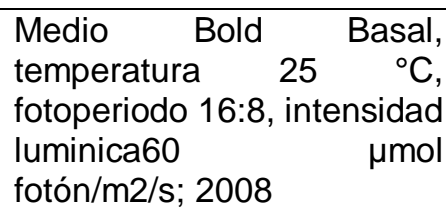 & $\begin{array}{c}24.5 \mathrm{mg} / \mathrm{g} \mathrm{de} \\
\text { peso seco }\end{array}$ \\
\hline 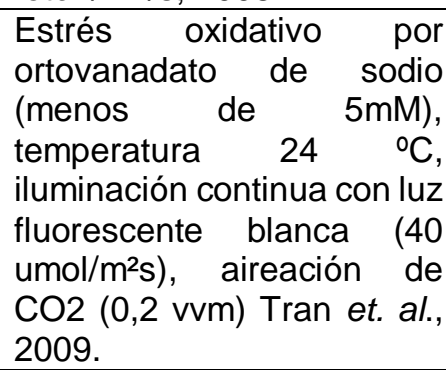 & $\begin{array}{c}10,7 \mathrm{mg} / \mathrm{g} \\
\text { biomasa }\end{array}$ \\
\hline $\begin{array}{l}\text { Bristol selectivo } \\
\text { Con agitación controlada de } \\
25 \circ \text { Cun fotoperiodo de } 12 \mathrm{~h} \text {. } \\
\text { luz-12 h. oscuridad } 0.25 \text {, } \\
0.50 \text {, } 0.75,1.0,1.25 \text { y } 1.5 \\
\text { g/L; Sosa, 2009; Ramírez, } \\
2013 \text {. }\end{array}$ & Acumulación \\
\hline $\begin{array}{l}\mathrm{F} / 2 \text { Sin Nitrógeno, } \mathrm{pH}, \\
8,3714-15^{\circ} \mathrm{C} \text {, Luz solar, luz } \\
\text { UV-vis, oscuridad; Sosa, } \\
2009\end{array}$ & Acumulación \\
\hline $\begin{array}{l}\mathrm{F} / 2 \text {,pH7-8,2, Temperatura } \\
14-15^{\circ} \mathrm{C} \text {, luz solar, luz UV- } \\
\text { vis, oscuridad; Sosa, } 2009\end{array}$ & Acumulación \\
\hline $\begin{array}{l}\mathrm{F} / 2, \quad \mathrm{pH} \quad 7,7-8,2, \\
\text { temperatura } 14-15^{\circ} \mathrm{C} \text {, luz } \\
\text { solar, luz UV-vis, oscuridad, } \\
\text { Sosa, } 2009 .\end{array}$ & Acumulación \\
\hline $\begin{array}{l}\text { Fortilizante folicular (QF), } \\
\mathrm{pH} 7,7-8,2 \text {, temperatura } 14 \\
-15{ }^{\circ} \mathrm{C} \text {, Luz solar, luz UV- } \\
\text { vis, oscuridad; Sosa, } 2009 .\end{array}$ & Acumulación \\
\hline $\begin{array}{l}\text { Temperatura } 25^{\circ} \mathrm{C}, \mathrm{pH} 8.0 \text {, } \\
\text { lluminación continua No se } \\
\text { dan concentraciones pero si } \\
\text { se usa Medio BG11 y RM } \\
\text { Variaron condiciones } \\
\text { autótrofas, heterotróficas y } \\
\text { mixotroficas. } 75 \text { mol } \\
\text { fotones; Imamoglu et al. } \\
2009 \text {. }\end{array}$ & Acumulación \\
\hline $\begin{array}{l}\text { Medio bristol, temperatura } \\
20^{\circ} \pm 2^{\circ} \mathrm{C}, \mathrm{pH} 6 \text {, lluminación } \\
\text { continua; Gómez et. al., } \\
2009 \text {. }\end{array}$ & $\begin{array}{c}58.42 \pm 4.0 \mathrm{pg} \\
\mathrm{cel}\end{array}$ \\
\hline $\begin{array}{l}\text { Medio M1, } \mathrm{pH} \quad 7.0, \\
\text { temperatura } 25 \pm 0,5{ }^{\circ} \mathrm{C} \text {, } \\
\text { lámparas fluorescentes } \\
\text { marca Sylvania Daylight } \\
\text { F48T12/D de } 39 \mathrm{~W} \text { con una }\end{array}$ & Acumulación \\
\hline
\end{tabular}

\begin{tabular}{|c|c|}
\hline $\begin{array}{l}\text { intensidad lumínica de } 5000 \\
\pm \quad 50 \quad \text { lux } 0.51 \% \quad \mathrm{CO}_{2} \\
\text { ausencia de nitrógeno; } \\
\text { Ramírez, 2013. }\end{array}$ & \\
\hline 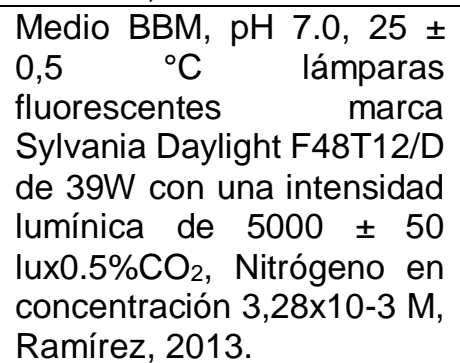 & Acumulación \\
\hline $\begin{array}{l}\text { Medio F1, } \mathrm{pH} \quad 7.0, \\
\text { Temperatura } 25 \pm 0,5{ }^{\circ} \mathrm{C} \text {, } \\
\text { lámparas fluorescentes } \\
\text { marca Sylvania Daylight } \\
\text { F48T12/D de } 39 \mathrm{~W} \text { con una } \\
\text { intensidad lumínica de } 5000 \\
\pm 50 \text { lux, } 0.51 \% \quad \mathrm{CO}_{2}, \\
\text { Concentración de nitrógeno } \\
3,28 \times 10-3 \quad \mathrm{M} ; \quad \text { Ramírez, } \\
2013 .\end{array}$ & Acumulación \\
\hline $\begin{array}{l}\text { Medio BG-11, } \mathrm{pH} 7.0, \\
\text { temperatura } 25 \pm 0,5{ }^{\circ} \mathrm{C} \text {, } \\
\text { lámparas fluorescentes } \\
\text { marca Sylvania Daylight } \\
\text { F48T12/D de } 39 W \\
\text { con una intensidad lumínica } \\
\text { de } 5000 \pm 50 \text { lux, } 0.51 \% \\
\mathrm{CO}_{2} \text { Concentración de } \\
\text { nitrógeno 3,28x10-3 M; } \\
\text { Ramírez, } 2013 \text {. }\end{array}$ & Acumulación \\
\hline $\begin{array}{l}\text { Medio HONG-KONG, } \\
\text { pH7.0, temperatura } 25 \pm \\
0,5^{\circ} \mathrm{C}, \quad \text { lámparas } \\
\text { fluorescentes marca } \\
\text { Sylvania Daylight F48T12/D } \\
\text { de } 39 \mathrm{~W} \text { con una intensidad } \\
\text { lumínica de } 5000 \pm 50 \text { lux, } \\
0.51 \% \mathrm{CO}_{2}, \text { ausencia de } \\
\text { nitrógeno; Ramírez, } 2013 \text {. }\end{array}$ & Acumulación \\
\hline $\begin{array}{l}\text { Medio M6, pH } 7.0, \\
\text { temperatura } 25 \pm 0,5 \quad{ }^{\circ} \mathrm{C} \\
\text { lámparas fluorescentes } \\
\text { marca Sylvania Daylight } \\
\text { F48T12/D de } 39 \mathrm{~W} \text { con una } \\
\text { intensidad lumínica de } 5000 \\
\pm 50 \text { lux, } 0.51 \% \quad \mathrm{CO}_{2 ;} ; \\
\text { Ramírez, } 2013 .\end{array}$ & Acumulación \\
\hline $\begin{array}{l}\text { Medio OHM, } \mathrm{pH} 7.0, \\
\text { temperatura } 25 \pm 0,5{ }^{\circ} \mathrm{C} \text {, } \\
\text { lámparas fluorescentes } \\
\text { marca Sylvania Daylight }\end{array}$ & Acumulación \\
\hline
\end{tabular}




\begin{tabular}{|c|c|}
\hline $\begin{array}{l}\text { F48T12/D de } 39 W \text { con una } \\
\text { intensidad lumínica de } 5000 \\
\pm \quad 50 \text { lux, } 0.51 \% \mathrm{CO}_{2}, \\
\text { concentración } \\
3,28 \times 10-3 \quad \mathrm{nitrógeno} \\
2013 .\end{array}$ & \\
\hline $\begin{array}{l}\text { Medio Z8, pH 7,0 } 25 \pm 0,5 \\
{ }^{\circ} \mathrm{C} \text {, lámparas fluorescentes } \\
\text { marca Sylvania Daylight } \\
\mathrm{F} 48 \mathrm{~T} 12 / \mathrm{D} \text { de } 39 \mathrm{~W} \text { con una } \\
\text { intensidad lumínica de } 5000 \\
\pm \quad 50 \text { lux } 0.51 \% \mathrm{CO}_{2}, \\
\text { Ausencia de nitrógeno; } \\
\text { Ramírez, } 2013 \text {. }\end{array}$ & Acumulación \\
\hline $\begin{array}{l}\text { Medio BBM, Temperatura } \\
30^{\circ} \mathrm{C}, \mathrm{pH} 5 \text {, lluminación } \\
\text { continua /acetato de sodio } \\
0,25 \% \text {; Hanan et al., } 2013\end{array}$ & Acumulación \\
\hline $\begin{array}{l}\text { Medio WC, temperatura } \\
23^{\circ} \mathrm{C}, \mathrm{pH} 7 \text {, fotoperiodo } \\
12 / 124 \% \text { de } \mathrm{CO}_{2} / 350 \\
\text { micromoles de fotones; } \\
\text { Nunes et al. } 2013 \text {. }\end{array}$ & $\begin{array}{l}17,66 \pm 1,22 \mathrm{mg} / \\
\mathrm{g}\end{array}$ \\
\hline $\begin{array}{l}\text { Medio BBM, temperatura } \\
24^{\circ} \mathrm{C} / \text { fotoperiodo12/12/Adic } \\
\text { ión de } 6 \text { concentraciones } \\
\text { distintas de vit B12, } \\
\text { intensidad lumínica } 60 \\
\mathrm{mmol} / \mathrm{m} 2 \text { / s; Li et al., } 2013 .\end{array}$ & $28,35 \mathrm{mg} / \mathrm{I}$ \\
\hline $\begin{array}{l}\text { Medio } \mathrm{BG} 11 \text {, temperatura } \\
20^{\circ} \mathrm{C}, \mathrm{pH} 7-8 \text {, iluminación } \\
\text { continua, } 1.5 \% \text { de } \mathrm{CO}_{2} \text {, } \\
\text { bajas concentraciones de } \\
\text { nitrógeno biomasa, } \\
\text { intensidad lumínica } 30 \mu \mathrm{mol} \\
\mathrm{m}-2 \mathrm{~s} \text {; Wang et al., } 2013 \text {. }\end{array}$ & $16.0 \mathrm{mg} \mathrm{L}-1 \mathrm{~d}-$ \\
\hline
\end{tabular}

\section{CONCLUSIÓN}

Se logró establecer que las condiciones de crecimiento adecuadas para la microalga $H$. pluvialis que llevan a una buena producción de astaxantina son: utilizar medios de cultivo como el BBM, OHM y BG11 bajo condiciones de $\mathrm{pH} 7$, fotoperiodo 20/4, temperatura 25 $\stackrel{\circ}{ } \mathrm{C}$, agitación continua, $\mathrm{CO}_{2} 5 \%$ e iluminación
La acumulación del pigmento en $H$. pluvialis ocurre durante la transformación de la microalga desde el estado vegetativo (fase verde) a aplanospora (fase roja) cuando cesa su crecimiento en la fase estacionaria.

De acuerdo a la revisión realizada los factores que inducen la acumulación de astaxantina pueden ser temperatura, intensidad lumínica, ciclos de luz/oscuridad, concentración de nutrientes, $\mathrm{pH}$, especies reactivas de oxígeno, sales y presencia de inhibidores de procesos metabólicos a diferente nivel, ya que esta microalga tiene sus dificultades en el momento de cultivo y de obtener el pigmento en cantidades de interés debido a su ciclo celular complejo. con lámparas fluorescentes blancas 40 umol $/ \mathrm{m}^{2} \mathrm{~s}$. 


\section{REFERENCIAS BIBLIOGRÁFICAS}

Ambati R, Phang S, Ravi S and Aswathanarayana R. (2014). Astaxanthin: Sources, Extraction, Stability,Biological Activities and Its Commercial Applications-A Review. Mar. Drugs. 2009,12: 128-152p.

Bosung Ku, Jeong J.C., Mijts B.N., SchmidtDannert C., and Dordick J.S..Preparation, Characterization, and Optimization of an In Vitro C30 Carotenoid Pathway. Applied and environmental microbiology, 2005. Vol. 71 No 11. 6578-6583p.

Cifuentes Ana, González Mariela, Vargas Silvia, Hoeneisen Maritza y González Nelsón. Optimization of biomass, total carotenoids and astaxanthin production in Haematococcus pluvialis Flotow strain Steptoe (Nevada, USA) under laboratory conditions. Biol Res 36, 2003. 343-357p.

Domínguez-Bocanegra A. R., Guerrero Legarreta I, Martinez Jeronimo $F$, Tomasini Campocosio A.Influence of environmental and nutritional factors in the production of astaxanthin from Haematococcus pluvialis. Bioresources Technolofy, 2004 Apr; 92 (2) : 209-14p.

Domínguez-Bocanegra R, Ponce-Noyola $T$, Torres-Muñoz J. Astaxanthin production by Phaffia rhodozyma and
Haematococcus pluvialis: a comparative study Appl Microbiol Biotechnol. 2007; 75: 783-791.

Eonseon J., Gyun lee Ch., and Polle J.. Secondary Carotenoid Accumulation in Haematococcus (Chlorophyceae): Biosynthesis, Regulation, and Biotechnology. Journal of of Microbiology and Biotechnology. 2006. 16: 821-83p.

Gómez L. Liliana, Menéndez Joaquin, Álvarez inaudis, Flores Ignacio. Efecto de diferentes protocolos de aplicación de un campo magnético (0.03T) sobre el crecimiento, viabilidad y composición pigmentaria de Haematococcus pluvialis Flotow en suficiencia y ausencia de nitrógeno. (2009). Biotecnología Vegetal, Vol. 9, No. 2: 105 - 117p.

Grünewald K.; Manfred E.;Hirschberg J Hage. C. Phytoene desaturase is localized exclusively in the Chloroplast and up-regulated and the mRNA level during accumulation of secondary carotenoids in Haematococcus pluvialis (Volvocales,Chlorophyciae). Plant Physiology.2000.Vol. 122.

Hanan N, Al-Shorgani N, Shukor H, Rahman N, KalilM. Pre-Optimization Conditions for Haematococcus pluvialis Growth. International Journal on advanced 
sciences, engineering and information technology. 2013. 2: 70-73p

Imamoğlu, Esra, Dalay Meltem Conk, Sukan Fazilet Vardar. Effect of different cultivation models on growth of Haematococcus pluvialis flotow, 2009, International journal of natural \& engineering sciences, vol. 3 issue 2,10p.

Kang C, Lee J, Park T,. Sim S. Complementary limiting factors of astaxanthin synthesis during photoautotrophic induction of Haematococcus pluvialis: $\mathrm{C} / \mathrm{N}$ ratio and light intensity. Appl Microbiol Biotechnol. 2007; 74: 987-994.

\section{Katsuda T.,Shimahara K.,Shiraishi}

H.,Yamagami K.,Ranbjar R.,Katoh S. Effect of flashing light from blue light emitting diodes on cell growth and Astaxanthin production of Haematococcus pluvialis. Journal of Bioscience and Bioengineering. 2006. Vol 102 No5.442-446p.

Lababpour A. Gyun Lee C. Simultaneous Measurements of Chlorophyll and Astaxanthin in Haematococcus pluvialis cells by first order derivative ultravioletvisible Spectrophotometry. Journal of Bioscience and Bioengineering.2013. 101:104-110p.
Labapour A. Shimahara K. Hada K. Kioui Y.Katsuda T.Katoh S. Fed-batch culture under illumination with blue light emitting diodes (LEDS) for Astaxanthin production by Haematococcus pluvialis.Journal of Bioscience and Bioengineering. 2005. Vol 100 No3. 339-342p.

Li Li Xin, Song Zhi-wei, Zhan You, Duan Shun-shan Zhao Qian-shen y Liu Yan. Effect of Vitamin-B12 and Vitamin-H on the Growth and Astaxanthin Content of Haematococcus pluvialis $\mathrm{CH}-1 \quad 1$. Advance Journal of Food Science and Technology, 2013.5(9): 1139-1142.

Meltem conk D., Imamoglu E., and Demirel $Z$. Agricultural fertilizers as economical alternative for cultivation of Haematococcus pluvialis.. J Microbiol. Biotechnol. 2007. Vol. 17(3). 393-397p.

Nunes, Moira, Vieira, Armando Augusto Henriques, Pinto, Ernani, Carneiro, Ronaldo Leal, \& Monteiro, Antonio Carlos. Carotenogênese em células de Haematococcus pluvialis induzidas pelos estresses luminoso

nutricional. Pesquisa Agropecuária Brasileira, 2013. 48(8), 825-832p.

Ramírez L. Daniel M. Evaluación del crecimiento y producción de astaxantina por Haematococcus pluvialis en un fotobiorreactor tipo airlift. Tesis de investigación. Universidad Nacional. 2013. 1-123p. 
Ranbjar R. Inoue R.,Katsuda T., Yamaji H.Katoh S. High efficiency production of Astaxanthin in an airlift photobioreactor. Journal of Bioscience and Bioengineering.2008. 2008.106: 204$207 p$

Rao R., Sarada A.R., Baskaran V., and Ravishankar G.A.. Identification of Carotenoids from Green Alga Haematococcus pluvialis by HPLC and LC-MS (APCl) and Their Antioxidant Properties. J. Microbiol. Biotechnol. 2009. Vol. 19(11). 1333-1341p.

Sosa L. Antonieta E. Cultivo de la microalga Haematococcus pluvialis, en lote $y$ fotobiorreactor para la produccion de carotenoides. Tesis. Universidad Autonoma Metropolitana, 2009. 1-40p.

Steinbremer J.; Linden H. Regulation of two carotenoid byosinthesis genes coding for phytoene synthase and carotenoid hidroxylase during stress-induced astaxanthin formation in the green alga. Haematococcus pluvialis. Plant Physiology, 2001.125, 810-817p.

Steinbrenner J. and Sandmann G.. Transformation of the Green Alga Haematococcus pluvialis with a Phytoene Desaturase for Accelerated Astaxanthin Biosynthesis. Applied and Environmental
Microbiology. 2006. Vol. 72 No. 12. $7477-$ $7484 p$.

Torzillo G., Tolga Göksan, Oya Isik and Gökpinar T.. Photon irradiance required to support optimal growth and interrelations between irradiance and pigment composition in the green alga Haematococcus pluvialis. Eur. J. Phycol.. 2005.Vol. 40(2). 233-240p.

Tran N.P., Park J K., Kim Z.H., and Gyun Lee Ch.. Influence of Sodium Orthovanadate on the Production of Astaxanthin from Green Algae Haematococcus lacustris Biotechnology and Bioprocess Engineering. 2009. Vol. 14. 322-329p.

Vidhyavathi Raman, Venkatachalam L., et al. Regulation of carotenoid byosinthetic genes expression and carotenoid accumulation in the green alga Haematococcus pluvialis under nutrient stress conditions. Journal of Experimental Botany 59, 2008.1409-1418p.

Wang B.; Zarca A. Astaxanthin accumulation in Haematococcus pluvialis (Chlorophyciae) as an active photoprotective process under high irradiance. Journal of Physiology.2003. Vol.39.1116-1124p.

Wang J, Sommerfeld M, Lu C and Hu Q. Combined effect of initial biomass density and nitrogen concentration on growth and 
astaxanthin production of Haematococcus pluvialis (Chlorophyta) in outdoor cultivation Algae 28, 2013. 193-202p.

Wang S.B. Milton M., Qiang Hu S. Proteomic analysis of molecular response to oxidative stress by the green alga Haematococcus pluvialis (Chlorophyceae). Planta. 2004. Vol. 220. 17-29p.

Yoshimura S.Ranbjar R.Inoue R.,Katsuda T.,Katoh S. Effective utilization of transmitted light for Astaxanthin production of Haematococcus pluvialis. Journal of Bioscience and Bioengineering. 2006. Vol 102 No2. 97-101p. 